Published in final edited form as:

Pediatr Nephrol. 2012 January ; 27(1): 115-121. doi:10.1007/s00467-011-1942-6.

\title{
Genetic basis of cystinosis in Turkish patients: a single-center experience
}

\author{
Rezan Topaloglu, \\ Dept of Pediatric Nephrology, Hacettepe University Faculty of Medicine, Ankara 06100, Turkey
}

Thierry Vilboux,

NIH, NHGRI, MGB, Bldg. 10, Rm. 10 C107, 10 Center Drive, Bethesda, MD 20892, USA

Turgay Coskun,

Department of Pediatric Metabolic Diseases, Hacettepe University Faculty of Medicine, Ankara 06100, Turkey

Fatih Ozaltin,

Dept of Pediatric Nephrology, Hacettepe University Faculty of Medicine, Ankara 06100, Turkey

Brad Tinloy,

NIH, NHGRI, MGB, Bldg. 10, Rm. 10 C107, 10 Center Drive, Bethesda, MD 20892, USA

Meral Gunay-Aygun,

NIH, NHGRI, MGB, Bldg. 10, Rm. 10 C107, 10 Center Drive, Bethesda, MD 20892, USA

Aysin Bakkaloglu,

Dept of Pediatric Nephrology, Hacettepe University Faculty of Medicine, Ankara 06100, Turkey

Nesrin Besbas,

Dept of Pediatric Nephrology, Hacettepe University Faculty of Medicine, Ankara 06100, Turkey

Lambert van den Heuvel,

Department of Pediatrics Academic Hospital Leuven, Herestraat 49, 3000 Leuven, Belgium

Robert Kleta, and

NIH, NHGRI, MGB, Bldg. 10, Rm. 10 C107, 10 Center Drive, Bethesda, MD 20892, USA

William A. Gahl

NIH, NHGRI, MGB, Bldg. 10, Rm. 10 C107, 10 Center Drive, Bethesda, MD 20892, USA

Rezan Topaloglu: rezantopaloglu@hacettepe.edu.tr

\section{Abstract}

We report the molecular findings for the CTNS gene in 12 Turkish cystinosis patients aged 7-29 years. All presented initially with severe failure to thrive, polyuria, and polydipsia. Cystinosis was diagnosed at age 1 month to 9 years. Seven patients reached end-stage renal failure at ages ranging from 6.5 to 15 years. Whereas three of the remaining five have renal Fanconi syndrome with proteinuria, two have had kidney failure of varying degrees. Molecular analyses involved an initial multiplex polymerase chain reaction (PCR) to determine the presence or absence of the $57-\mathrm{kb}$ northern European founder deletion in CTNS, followed by sequencing of the ten coding exons of CTNS. Comprehensive mutation analysis verified that none of the 12 patients carried the common $57-\mathrm{kb}$ deletion. We identified four previously reported nucleotide variations associated with 
cystinosis and five new variants: a 10-kb deletion, three missense variants, and a nucleotide substitution in a potential branch point site of intron 4. This study is the first molecular analysis of Turkish cystinosis patients and provides guidance for the molecular diagnosis of cystinosis in this population.

\section{Keywords}

Cystinosis; CTNS gene; Turkish patients; Genetic basis; New mutations

\section{Introduction}

Cystinosis (CTNS; OMIM 219800) is an autosomal recessive disease caused by mutations of the CTNS gene (OMIM 606272; GenBank NM_004937.2) located on chromosome 17p13. CTNS codes for cystinosin, a protein that facilitates cystine egress from lysosomes. Defective lysosomal transport leads to widespread accumulation and crystallization of cystine in many organs, particularly the kidney, cornea, bone marrow, thyroid, lymph nodes, liver, and spleen [1-3]. The most common and severe phenotype is infantile nephropathic cystinosis in which renal signs and symptoms predominate; patients develop renal Fanconi syndrome in infancy and renal failure in the first decade. Other organs frequently affected are the cornea, causing painful photophobia, and the thyroid, associated with hypothyroidism. Myopathy, swallowing, pulmonary dysfunction, diabetes, male hypogonadism, and central nervous system involvement are seen in adolescents and young adults with nephropathic cystinosis [1,4]. A milder cystinosis phenotype presents in adolescence with more slowly progressive renal failure and ocular disease, and an adult form (i.e., ocular) presents with only corneal disease $[1,5]$.

Management and treatment of infantile nephropathic cystinosis includes supportive treatment to maintain fluid and electrolyte balance in infancy and early childhood. Early diagnosis and specific treatment with orally administered cysteamine delays the onset of renal failure and prevents the occurrence of other serious organ involvement $[6,7]$. Cysteamine eye drops may lessen visual symptoms by dissolving the corneal cystine crystals. Hemodialysis (HD), continuous ambulatory peritoneal dialysis (CAPD), and renal transplantation (Tx) are associated with increased survival rates in patients who progressed to end-stage renal failure (ESRF). Cystine accumulation has been reported in transplanted kidneys but with no clinical manifestations, as it reflects invasion of host cells containing cystine. However, after renal Tx, extrarenal manifestations of cystinosis continue to occur [1].

The $C T N S$ gene consists of 12 exons; numbers 3-12 are coding exons. CTNS mutations result in either complete abolition of, or reduced cystine transport [1,5]. Many different mutations have been identified throughout the entire gene in European and American cystinosis patients [8-18]. The most common mutation is a large 57-kb deletion [11, 13-16]. Here we report $C T N S$ mutation analysis of 12 Turkish patients with cystinosis, representing the first such molecular diagnosis of cystinosis in this population.

\section{Materials and methods}

\section{Patients}

We studied 12 Turkish patients who were diagnosed with cystinosis and followed at Hacettepe University Faculty of Medicine Children's Hospital Department of Pediatric Nephrology and Metabolic Diseases Unit. Informed consent was obtained from the patients 
and/or their parents. Blood samples were collected from all patients; DNA was extracted from leukocytes, as previously described [19].

\section{Molecular analysis}

The 57-kb deletion was tested using the method previously described [20]. Primers were designed to amplify the ten coding exons of $C T N S$, including their intronic boundaries (primer sequences available on request). Standard polymerase chain reaction (PCR) amplification procedures were employed. All PCR amplified products were directly sequenced using the Big Dye 3 Terminator chemistry (Applied Biosystems) employing an ABI 3130xl genetic analyzer (Applied Biosystems). Data were analyzed using Sequencher 4.8 (Gene Codes Corporation).

\section{Mutation prediction analysis}

The effect of missense variants and potential splice-site variants was evaluated, as described previously [21].

\section{Results}

Twelve patients (seven male; five female) from ten families were evaluated. Patients' ages ranged from 7 to 29 years; age at diagnosis was 1 month to 9 years. Consanguinity was reported for six families, and in two families there were two affected siblings. All patients were put on cysteamine bitartrate treatment at diagnosis. Six patients reached ESRF; one is receiving $\mathrm{PD}$, three are on $\mathrm{HD}$, and two have undergone renal Tx. Of the remaining six patients, three had varying degree of renal failure and three had proteinuria at the time of the study (Table 1). Before sequencing, all patients were tested for the 57-kb deletion and it was not found in any of them. Analysis of the ten coding exons of CTNS revealed the presence of nucleotide variations in all patients. Thirteen different variants (Table 1) were identified in the 12 patients; four were already referenced in the dbSNP (http://www.ncbi.nlm.nih.gov/ $\mathrm{snp} /)(\mathrm{rs} 161400, \mathrm{rs} 1800528, \mathrm{rs} 222753$, and rs76153698). The others were one deletion (10 $\mathrm{kb}$ ), one small deletion (4 bp), four missense variants, two potential splice-site modifications, and a nucleotide substitution in a potential branch-point site of intron 4 .

Newly identified missense variations c. $451 \mathrm{~A}>\mathrm{G}, \mathrm{c} .518 \mathrm{~A}>\mathrm{G}$, and c.470 G>A were searched in 100 healthy Turkish controls by direct sequencing; none of these three variants was found in healthy Turkish controls (Figs. 1-3). We also demonstrated segregation in two families in which DNA was available (Figs. 2 and 3).

The potential effects of the nucleotide changes on cystinosin are listed in Table 2.

\section{Discussion}

The diagnosis of cystinosis is confirmed by demonstrating markedly elevated cystine levels in polymorphonuclear leucocytes [1,22]. Detection of corneal crystals by slit-lamp examination is virtually diagnostic of cystinosis in patients with Fanconi syndrome. However, corneal crystals may not develop until 1-1.5 years of age, so this finding is not sensitive for early diagnosis that, along with diligent compliance with cystine-depleting treatment, determines prognosis. Another determinant is genetic predisposition. Infantile nephropathic cystinosis is associated with two severe CTNS mutations, including deletions, insertions, nonsense, missense, and splicing mutations, that cause complete abolition of cystine transport. The adolescent and ocular forms generally have one severe and one mild mutation, leading to reduced cystine transport [1, 5, 23]. Molecular studies have revealed that $50-60 \%$ of northern European and North American patients with cystinosis have a common 57-kb deletion that affects the first ten exons of the CTNS gene, consistent with a 
founder effect $[11,13-16]$. On the other hand, the 57-kb deletion has been detected with a much lower frequency in Italians, French Canadians, and Mexicans [12, 18, 20]; the carrier rate was only $17 \%$ in Italians. HGMD (http://www.hgmd.cf.ac.uk/ac/index.php) describes approximately 100 mutations in the $C T N S$ gene.

This report represents the first description of the genetic basis of cystinosis in Turkish patients. Our data reveal that the Turkish cystinosis population has novel genetic characteristics. Specifically, we did not detect the northern European 57-kb deletion in any Turkish patient. Of 13 different variants identified, five were novel, such as three missense variants [c.518A>G (p.Y173C), c.451A>G (p.R151G), c.470 G>A (p.G157D)], a new gross deletion (c.62-1083_551del10217bp), removing nucleotides between intron 3 and exon 8, and the sequence variation in a potential consensus branch point sequence (c.141-22a $>\mathrm{g}$ ). Although no functional studies have been performed, the amino-acid changes p.Y173C, p.R151G, and p.G157D are very likely to be causative mutations based on formal criteria, evolutionary conservation, and absence in healthy controls, as well as prediction software (Figs. 1- 3 and Table 2). The sequence variation c.141-22a>g may disturb a consensus sequence for a branch point and interfere with correct or efficient splicing [24]. Regular software to predict efficiency of RNA splicing does not exist for branch sites to our knowledge. Confirmation that this variant has an effect on splicing analysis on the RNA level is required. [25].

Of the eight previously reported variants, four had been associated with cystinosis [8-10, $26]$ and four were referenced in the dbSNP. The variant c.140+1 G>T has been described as $479+1 \mathrm{G}>\mathrm{T}$ according to old nomenclature; it affects a canonical splice site and results in loss of cystinosin function [8]. The variant c.1015 G>A (p.G339R) abolishes cystine transport in an in vitro functional model $[9,10]$. The variant 4-bp deletion c.18_21del GACT (p.T7FX7) was described by Town et al. [8] and reported in different populations with cystinosis. The variant c.681 G>A (p.E227E), described in an Arabic population, is located in an exon-intron boundary and disturbs normal splicing [26].

In our study, the most common variants identified were rs 222753 at around $45 \%$, as described in the dbSNP; and c.681 G>A; pE227E at around 29\%, as a disease-causing variant [26]. Interestingly, the consanguinity of TR2, TR3, TR6, TR7, TR11, and TR12 is supported by the presence of homozygous variants. However, for patients TR4, TR5, and TR37, heterozygous variants are present despite parental consanguinity. Clinically, the patient homozygous for the 10-kb deletion (TR3) followed a severe course and progressed to ESRF at 10 years of age. Patients TR6 and TR7, homozygous for the new missense variants c.518A $>\mathrm{G}$ (p.Y173C) and c.451A>G (p.R151G), both had moderate disease courses, reaching adolescence with proteinuria but no renal failure. Patient TR121, homozygous for the known variant c. $140+1 \mathrm{G}>\mathrm{T}$ that affects a canonical splice site, had a severe disease course [8]. The siblings (TR11 and TR12) homozygous for c.681 G>A (pE227E) exhibited a severe disease course but were the most noncompliant patients, which might be related to disease course. TR1, compound heterozygous for a new missense variant c.451 $\mathrm{A}>\mathrm{G}$ (p.R151G) and a known missense variant c.1015 G>A (p.G339R), had a mild disease course, with good compliance and largely intact renal function at age 12. On the other hand, TR36, compound heterozygous for the c.681 G>A (p.E227E) and the same known missense variant (c.1015 G>A; p.G339R), is noncompliant and has experienced severe disease, with ESRF at approximately 10 years of age; she was recently transplanted with her mother's kidney.

The discrepancy in disease course in patients TR2 and TR 37 could be due to the different variants in the second allele as well as compliance to cysteamine treatment. TR2, homozygous for a known 4-bp deletion c.18_21delGACT; p.T7FX7, has severe disease with 
liver involvement. This deletion has been found in different populations and with widely variable disease severities $[8,9]$. TR37 was compound heterozygous for a new missense mutation c. $470 \mathrm{G}>$ A (p.G157D) inherited from her father (Fig. 3b) and a known deletion c. 18_21delGACT (p.T7FX7) inherited from her mother. The patient had a fairly good course but progressed to end-stage renal disease (ESRD) at 16 years. Siblings TR4 and TR5 were compound heterozygous for a known splice-site variant c.681 G>A (p.E227E) and a new variant c.141-22a>g. These siblings had a completely different courses. Although TR4 was diagnosed at age 13 months, he was lost to follow-up for a long period and progressed to ESRD at age 9.5 years. On the other hand, TR5 had adequate management and follow-up and did not required renal replacement treatment as of age 17.5 years. These observations confirm once again the key role of early diagnosis and adequate cysteamine treatment in the course of cystinosis. From the genetic point of view, our findings support previous data on genotype-phenotype correlations of cystinosis patients [1, 5, 23].

In summary, we show that Turkish cystinosis patients have different disease-causing variants of $C T N S$ compared with European and North American patients. In light of our results, it seems that toward to Mediterranean region, the frequency of the 57-kb deletion decreases. In northern Europe and North American studies, the frequency approximates 50$70 \%$, whereas it decreases to $17 \%$ in Italy [18] and is absent in this Turkish cohort. Our data once more confirm the wide mutational spectrum of the $C T N S$ gene. Data provide a basis for further molecular diagnosis in the Turkish population.

\section{Acknowledgments}

We gratefully acknowledge Prof. Dr. Elena Levtchenko for her advice and help in completing the genetic analysis and the critical reading of the manuscript. Hacettepe University Nephrogenetics Laboratory was established by Hacettepe University Infrastructure Project (Project No: 06A101008)

\section{References}

1. Gahl WA, Thoene JG, Schneider JA. Cystinosis. N Engl J Med. 2002; 347:111-121. [PubMed: 12110740]

2. Schneider JA, Rosenbloom FM, Bradley KH, Seegmiller JE. Increased free-cystine content of fibroblasts cultured from patients with cystinosis. Biochem Biophys Res Commun. 1967; 29:527531. [PubMed: 16496530]

3. Gahl WA, Bashan N, Tietze F, Bernardini I, Schulman JD. Cystine transport is defective in isolated leukocyte lysosomes from patients with cystinosis. Science. 1982; 217:1263-1265. [PubMed: 7112129]

4. Sonies BC, Almajid P, Kleta R, Bernardini I, Gahl WA. Swallowing dysfunction in 101 patients with nephropathic cystinosis: benefit of long-term cysteamine therapy. Medicine (Baltimore). 2005; 84:137-146. [PubMed: 15879904]

5. Kalatzis V, Antignac C. New aspects of the pathogenesis of cystinosis. Pediatr Nephrol. 2003; 18:207-215. [PubMed: 12644911]

6. Gahl WA, Balog JZ, Kleta R. Nephropathic cystinosis in adults: natural history and effects of oral cysteamine therapy. Ann Intern Med. 2007; 147:242-250. [PubMed: 17709758]

7. Gahl WA, Reed GF, Thoene JG, Schulman JD, Rizzo WB, Jonas AJ, Denman DW, Schlesselman JJ, Corden BJ, Schneider JA. Cysteamine therapy for children with nephropathic cystinosis. N Engl J Med. 1987; 316:971-977. [PubMed: 3550461]

8. Town M, Jean G, Cherqui S, Attard M, Forestier L, Whitmore SA, Callen DF, Gribouval O, Broyer M, Bates GP, van't Hoff W, Antignac C. A novel gene encoding an integral membrane protein is mutated in nephropathic cystinosis. Nat Genet. 1998; 18:319-324. [PubMed: 9537412]

9. Shotelersuk V, Larson D, Anikster Y, McDowell G, Lemons R, Bernardini I, Guo J, Thoene J, Gahl WA. CTNS mutations in an American-based population of cystinosis patients. Am J Hum Genet. 1998; 63:1352-1362. [PubMed: 9792862] 
10. Kaletzis V, Nevo N, Cherqui S, Gasnier B, Antignac C. Molecular pathogenesis of cystinosis: effect of CTNS mutations on the transport activity and subcellular localization of cystinosin. Hum Mol Genet. 2004; 13:1361-1371. [PubMed: 15128704]

11. Anikster Y, Shotelersuk V, Gahl WA. CTNS mutations in patients with cystinosis. Hum Mutat. 1999; 14:454-458. [PubMed: 10571941]

12. McGowan-Jordan J, Stoddard K, Podolsky L, Orrbine E, McLaine P, Town M, Goodyer P, MacKenzie A, Heick H. Molecular analysis of cystinosis: probable Irish origin of the most common French Canadian mutation. Eur J Hum Genet. 1999; 7:671-678. [PubMed: 10482956]

13. Heil SG, Levtchenko E, Monnens LA, Trijbels FJ, Van der Put NM, Blom HJ. The molecular basis of Dutch infantile nephropathic cystinosis. Nephron. 2001; 89:50-55. [PubMed: 11528232]

14. Kalatzis V, Cherqui S, Jean G, Cordier B, Cochat P, Broyer M, Antignac C. Characterization of a putative founder mutation that accounts for the high incidence of cystinosis in Brittany. $\mathrm{J}$ Am Soc Nephrol. 2001; 12:2170-2174. [PubMed: 11562417]

15. Kiehntopf M, Schickel J, Gonne B, Koch HG, Superti-Furga A, Steinmann B, Deufel T, Harms E. Analysis of the CTNS gene in patients of German and Swiss origin with nephropathic cystinosis. Hum Mutat. 2002; 20:237. [PubMed: 12204010]

16. Kleta R, Anikster Y, Lucero C, Shotelersuk V, Huizing M, Bernardini I, Park M, Thoene J, Schneider J, Gahl WA. CTNS mutations in African American patients with cystinosis. Mol Genet Metab. 2001; 74:332-337. [PubMed: 11708862]

17. Kalatzis V, Cohen-Solal L, Cordier B, Frishberg Y, Kemper M, Nuutinen EM, Legrand E, Cochat $\mathrm{P}$, Antignac C. Identification of 14 novel CTNS mutations and characterization of seven splice site mutations associated with cystinosis. Hum Mutat. 2002; 20:439-446. [PubMed: 12442267]

18. Mason S, Pepe G, Dall'Amico R, Tartaglia S, Casciani S, Greco M, Bencivenga P, Murer L, Rizzoni G, Tenconi R, Clementi M. Mutational spectrum of the CTNS gene in Italy. Eur J Hum Genet. 2003; 11:503-508. [PubMed: 12825071]

19. Huizing M, Anikster Y, Fitzpatrick DL, Jeong AB, D'Souza M, Rausche M, Toro JR, KaiserKupfer MI, White JG, Gahl WA. Hermansky-Pudlak syndrome type 3 in Ashkenazi Jews and other non-Puerto Rican patients with hypopigmentation and platelet storage-pool deficiency. Am J Hum Genet. 2001; 69:1022-1032. [PubMed: 11590544]

20. Anikster Y, Lucero C, Touchman JW, Huizing M, McDowell G, Shotelersuk V, Green ED, Gahl WA. Identification and detection of the common $65-\mathrm{kb}$ deletion breakpoint in the nephropathic cystinosis gene (CTNS). Mol Genet Metab. 1999; 66:111-116. [PubMed: 10068513]

21. Vilboux T, Kayser M, Introne W, Suwannarat P, Bernardini I, Fischer R, O’Brien K, Kleta R, Huizing M, Gahl WA. Mutation spectrum of homogentisic acid oxidase (HGD) in alkaptonuria. Hum Mutat. 2009; 30:1611-1619. [PubMed: 19862842]

22. Attard M, Jean G, Forestier L, Cherqui S, van't Hoff W, Broyer M, Antignac C, Town M. Severity of phenotype in cystinosis varies with mutations in the CTNS gene: predicted effect on the model of cystinosin. Hum Mol Genet. 1999; 8:2507-2514. [PubMed: 10556299]

23. Alcantara-Ortigoza MA, Belmont-Martinez L, Vela-Amieva M, Gonzalez-Del Angel A. Analysis of the CTNS gene in nephropathic cystinosis Mexican patients: report of four novel mutations and identification of a false positive 57-kb deletion genotype with LDM-2/exon 4 multiplex PCR assay. Genet Test. 2008; 12:409-414. [PubMed: 18752449]

24. Baralle D, Baralle M. Splicing in action: assessing disease causing sequence changes. J Med Genet. 2005; 42:737-748. [PubMed: 16199547]

25. Taranta A, Wilmer MJ, van den Heuvel LP, Bencivengo F, Bellomo F, Levtchenko EN, Emma F. Analysis of CTNS gene transcripts in nephropathic cystinosis. Pediatr Nephrol. 2010; 25:12631267. [PubMed: 20352457]

26. Aldahmesh MA, Humeidan A, Almojalli HA, Khan AO, Rajab M, AL-A AA, Meyer BF, Alkuraya FS. Characterization of CTNS mutations in Arab patients with cystinosis. Ophthalmic Genet. 2009; 30:185-189. [PubMed: 19852576] 


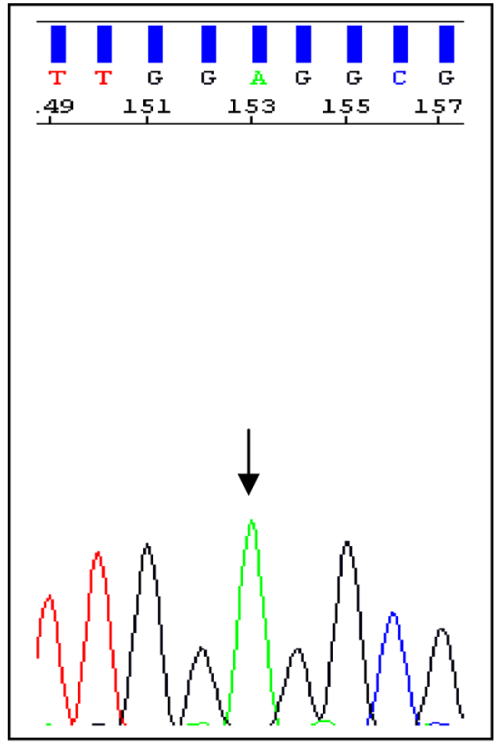

a

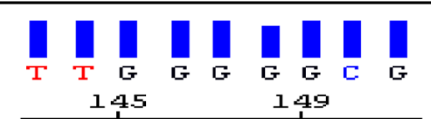

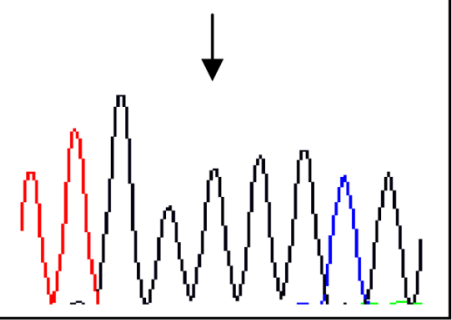

b

Fig. 1.

Sequence electropherograms of exon 5 of the $C T N S$ gene for c.451 A>G variant. The wildtype sequence in a healthy control is shown in the left electropherogram (a, arrow); the affected individual has a homozygous c.451 A>G variation (b, arrow) 


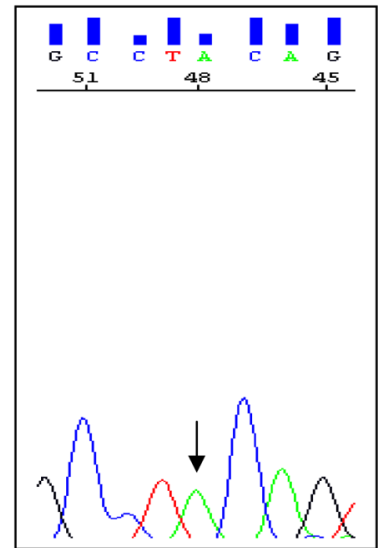

a

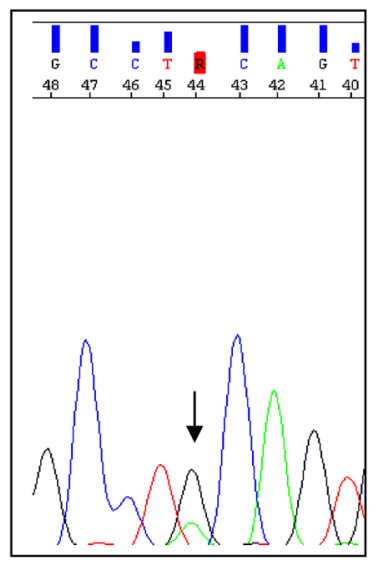

b

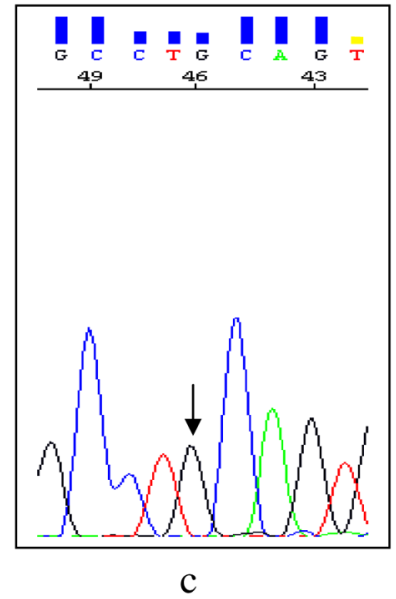

c

Fig. 2.

Sequence electropherograms of exon 6 of the $C T N S$ gene for c.518A $>\mathrm{G}$ variation. The wildtype sequence in a healthy control is shown in the left electropherogram (a, arrow). Parents are heterozygous (b, arrow); the affected individual has a homozygous c.518A $>\mathrm{G}$ variation (c, arrow) 


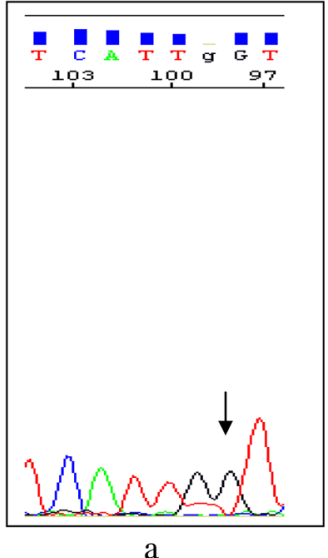

a

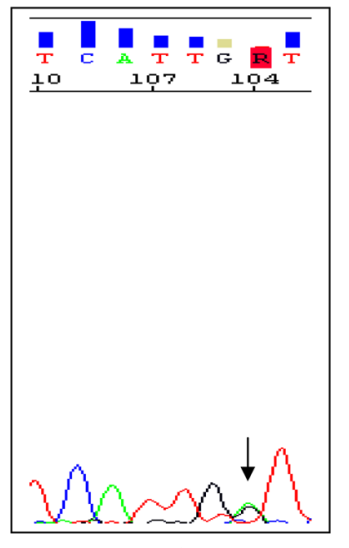

b

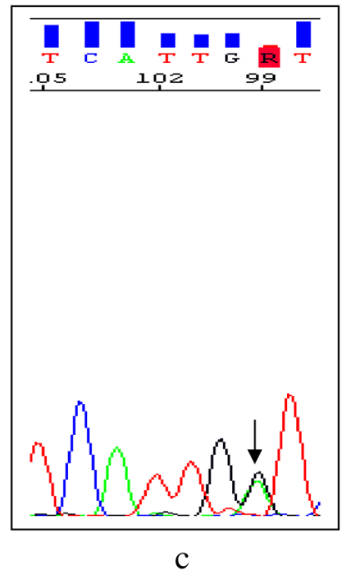

Fig. 3.

Sequence electropherograms of exon 6 of the $C T N S$ gene for c.470 G>A variation. The wild-type sequence in a healthy control is shown in the left electropherogram (a, arrow); the father is heterozygous (b, arrow); the affected individual has a heterozygous c.470 G>A variation (c, arrow) 


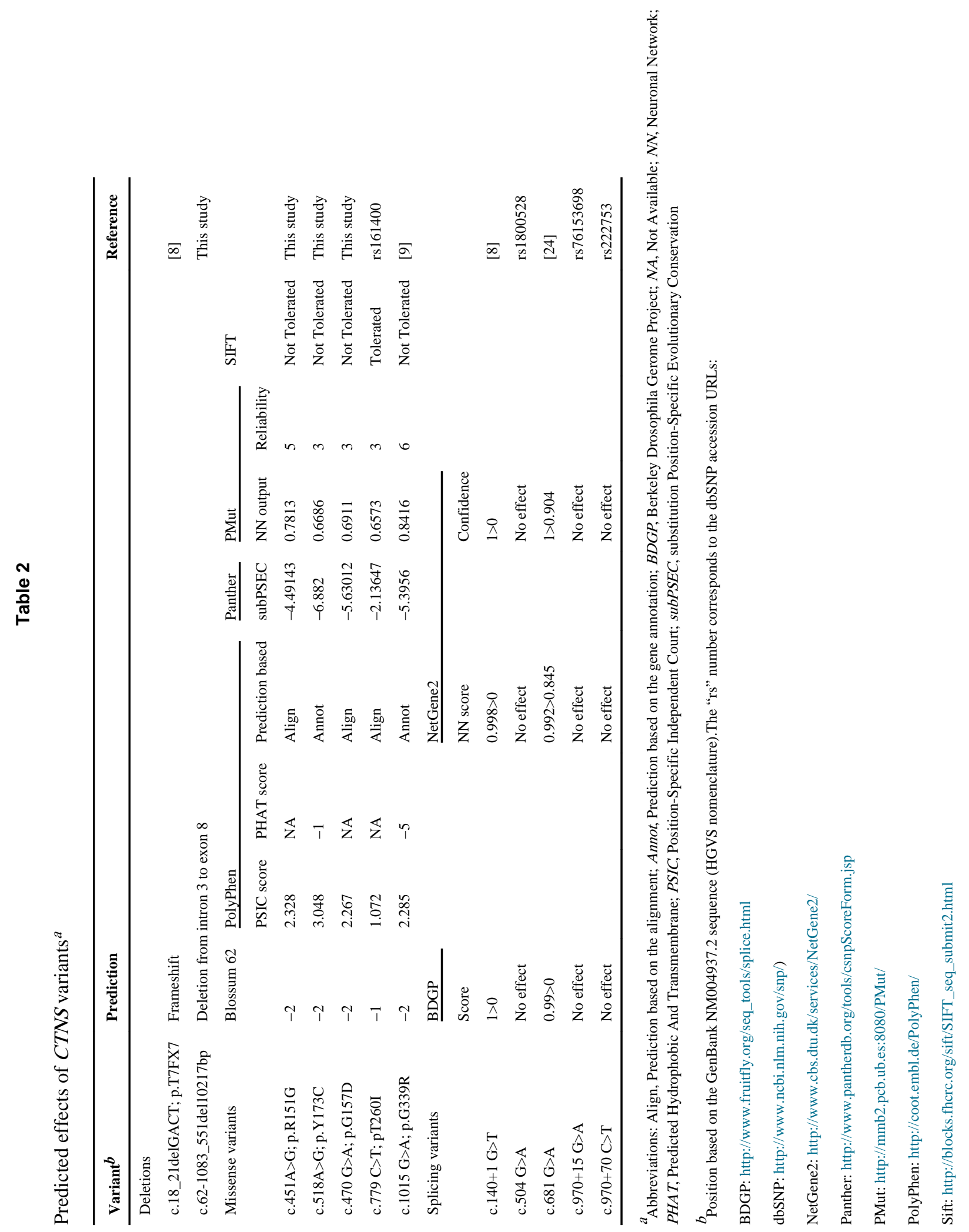

Pediatr Nephrol. Author manuscript; available in PMC 2013 January 01. 